\title{
HFR1 is targeted by COP1 E3 ligase for post-translational proteolysis during phytochrome A signaling
}

\author{
In-Cheol Jang, ${ }^{1}$ Jun-Yi Yang, ${ }^{1}$ Hak Soo Seo, and Nam-Hai Chua ${ }^{2}$ \\ Laboratory of Plant Molecular Biology, Rockefeller University, New York, New York 10021, USA
}

\begin{abstract}
Upon activation by far-red light, phytochrome A signals are transduced through several pathways to promote photomorphogenesis. The COP1 E3 ligase represses photomorphogenesis in part by targeting transcription activators such as LAF1 and HY5 for destruction. Another positive regulator of photomorphogenesis is HFR1, a basic helix-loop-helix (bHLH) transcription factor. Here, we show that HFR1 colocalizes with COP1 in nuclear bodies, and that the HFR1 N-terminal region (amino acids 1-131) interacts with the COP1 WD40 domain. HFR1 $(\Delta N)$, an HFR1 mutant lacking the two $N$-terminal, COP1-interacting motifs, still localizes in nuclear bodies and retains weak affinity for COP1. Both HFR1 and HFR1 $(\Delta N)$ can be ubiquitinated by COP1, although with different efficiencies. Expression of $35 S-H F R 1(\Delta N)$ in wild-type plants confers greater hypersensitivity to FR than 35S-HFR1 expression, and only seedlings expressing 35S-HFR1( $\Delta N)$ display constitutive photomorphogenesis. These phenotypic differences can be attributed to the instability of HFR1 compared with HFR1 $(\Delta N)$. In transgenic plants, HFR1 levels are significantly elevated upon induced expression of a dominant-negative COP1 mutant that interferes with endogenous COP1 E3 activity. Moreover, induced expression of wild-type COP1 in transgenic plants accelerates post-translational degradation of HFR1 under FR light. Taken together, our results show that HFR1 is ubiquitinated by COP1 E3 ligase and marked for post-translational degradation during photomorphogenesis.
\end{abstract}

[Keywords: Photomorphogenesis; light signaling; ubiquitination; proteasomal degradation]

Received August 6, 2004; revised version accepted January 14, 2005.

Plants depend on light not only as a source of energy for photosynthesis, but also for the timing of important developmental processes such as seed germination, stem elongation, and the transition to reproductive growth. A network of components transduces signals elicited by light of different wavelengths and intensities, and amplifies and coordinates these light signals with other environmental cues to execute appropriate physiological and developmental changes (Nagy and Schafer 2002; Quail 2002). Plants perceive light of different wavelengths through photoreceptors, such as the red/far-red light receptors known as phytochromes. Among the five members of Arabidopsis phytochrome (phy) protein family, phyA is the only one that is activated by far-red (FR) light, and is involved mainly in the regulation of seedling de-etiolation (Neff et al. 2000). Despite significant recent progress, only a few of the signaling components that transduce phyA signals have been identified, and the functional relationships between these components are not well understood.

\footnotetext{
${ }^{1}$ These authors contributed equally to this work.

${ }^{2}$ Corresponding author.

E-Mail chua@mail.rockefeller.edu; Fax (212) 327-8327.

Article and publication are at http://www.genesdev.org/cgi/doi/10.1101/ gad.1247205
}

Several approaches have been used to investigate phyA signal-transduction components. Under FR light, which inhibits cell elongation, wild-type seedlings have short hypocotyls. Arabidopsis mutants blocked in transmission of phyA signals display long hypocotyls under the same conditions. Several such mutants (hy5, fhy1, fhy3, fin219, far1, pat1, rsf1/hfr1/rep1, laf1, laf3, and laf6) have been characterized and their wild-type gene products have been shown to localize in the cytoplasm, nucleus, and chloroplasts (Oyama et al. 1997; Hudson et al. 1999; Bolle et al. 2000; Fairchild et al. 2000; Fankhauser and Chory 2000; Hsieh et al. 2000; Soh et al. 2000; Ballesteros et al. 2001; Desnos et al. 2001; Møller et al. 2001; Zeidler et al. 2001; Wang and Deng 2002; Hare et al. 2003a). This indicates the complexity of phyA responses. Among these signaling components, only LAF1 (Ballesteros et al. 2001), HY5 (Oyama et al. 1997), and HFR1 (Fairchild et al. 2000; Fankhauser and Chory 2000; Soh et al. 2000) are known to be transcription factors. These factors likely regulate expression of a subset of phyA responsive target genes (e.g., genes required for chloroplast development and photosynthesis) or genes encoding other signaling intermediates required for complete responsiveness to activated phyA.

In additional to transcription control, recent evidence 
suggests that regulated proteolysis also plays an important role in phyA signaling (cf. Hare et al. 2003b). Both HY5 (Osterlund et al. 2000) and LAF1 (Seo et al. 2003) have been shown to interact with the nuclear protein CONSTITUTIVE PHOTOMORPHOGENESIS (COP) 1 that acts to repress photomorphogenesis in the dark (Deng et al. 1992). COP1 is a RING motif protein that contains a coil-coiled domain and a WD40 repeats. This RING protein is able to self-ubiquitinate as well as ubiquitinate LAF1 and HY5 in vitro (Saijo et al. 2003; Seo et al. 2003). In transgenic plants expressing 35S::LAF1 and inducible $C O P 1$, there is an inverse relationship between the levels of LAF1 and COP1; increases in COP1 abundance lead to decreases in LAF1 levels (Seo et al. 2003). The decrease in LAF1 abundance associated with induction of COP1 can be blocked by proteasome inhibitors, implicating $26 \mathrm{~S}$ proteasomes as direct participants in LAF1 destruction (Seo et al. 2003). These results suggest that in darkness, nuclear COP1 represses photomorphogenesis by targeting a subset of transcription activators, including LAF1 and HY5, for degradation. In FR light, nuclear depletion of COP1 (von Arnim and Deng 1994) would conceivably permit the accumulation of LAF1 and HY5 to activate target genes.

The strong constitutive photomorphogenesis phenotype of cop 1 mutants in the dark suggests that additional transcription factors involved in phyA signaling may also be regulated by this E3 ligase. Here, we show that HFR1 can serve as a substrate of COP1 E3 ligase in vitro. Moreover, this transcription factor colocalizes with COP1 in nuclear bodies, and its levels can be increased by inducible expression of a dominant-negative (DN) COP1 mutant, which blocks endogenous COP1 E3 activity. Our results provide evidence for post-translational regulation of HFR1 by COP1.

\section{Results}

\section{Colocalization of HFR 1 and COP1 in nuclear bodies}

Although HFR1 was characterized several years ago (Fairchild et al. 2000; Fankhauser and Chory 2000; Soh et al. 2000), the subcellular location of its protein product has never been examined. To investigate this issue, we transiently expressed in onion epidermal cells a gene encoding an HFR1-YFP fusion protein. Genes encoding CFP and YFP alone were used as controls. Figure 1 shows that CFP and YFP were distributed throughout the cytosol as well as the nucleus. In contrast, HFR1-YFP was found only in nuclear bodies, and this specific localization was not altered by coexpression of CFP. Results from similar experiments confirmed previous observations that COP1 localized in the cytoplasm as well as in nuclear bodies (von Arnim and Deng 1994; Seo et al. 2003). Coexpression of HFR1-YFP and CFP-COP1 demonstrated that in the majority of the cases, the two proteins localized in the same nuclear bodies. However, it is not known whether the colocalization of HFR1/COP1 in nuclear bodies may be cell-type specific in Arabidopsis.

Interaction of several phyA signal transducers, e.g.,
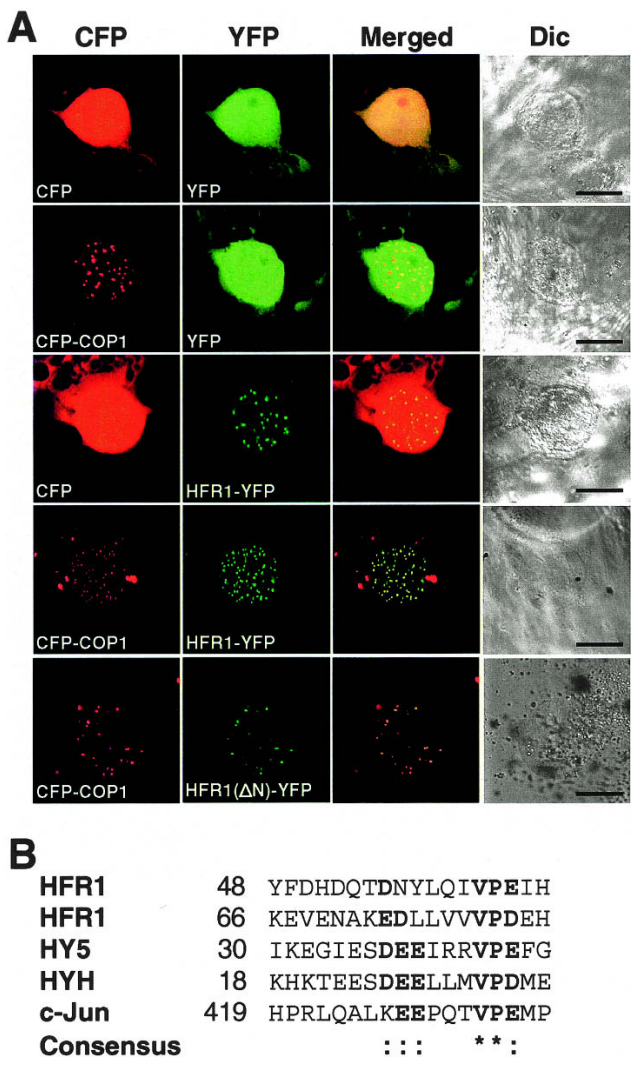

Figure 1. Colocalization of $\operatorname{HFR} 1$ or $\operatorname{HFR} 1(\Delta N)$ with COP1 in nuclear bodies and sequence comparison of putative COP1-interacting domains of several proteins. (A) Onion peels were cobombarded with equal amounts of the DNA constructs indicated. Epidermal cells were imaged using CFP and YFP channels of a confocal microscope. Bar, $20 \mu \mathrm{m}$. (CFP) CFP channel image; (YFP) YFP channel image; (Merged) merged image between CFP and YFP; (Dic) differential interference contrast in light microscope mode. $(B)$ Amino acid alignment of putative COP1-interacting domains in Arabidopsis HFR1, HY5, and HYH, as well as human c-Jun (Holm et al. 2001a,b; Bianchi et al. 2003). Colons and asterisks mark conserved acidic amino acids and the core sequence V-P-E/D, respectively.

HY5 with COP1, are known to be mediated by a COP1interacting domain (Hardtke et al. 2000). Two copies of this consensus sequence motif are found in the HFR 1 $\mathrm{N}$-terminal region (amino acids 48-83; Fig. 1B). To investigate the function of this motif, we deleted the first N-terminal 101 amino acids from wild-type HFR1 to give $\operatorname{HFR} 1(\Delta \mathrm{N})$. The $\operatorname{HFR} 1(\Delta \mathrm{N})$-YFP fusion protein, which lacks the putative COP1-interacting domain still colocalized with CFP-COP1 in nuclear bodies, suggesting that the $\mathrm{N}$-terminal domain is not required for the subnuclear localization.

\section{Interaction domains of HFR 1 and COP1}

The colocalization of HFR1 and COP1 suggested that the two proteins may interact. Using yeast two-hybrid assays, full-length HFR1 was found to interact with fulllength COP1 (Fig. 2). Deletion experiments identified 
the $\mathrm{N}$-terminal region (amino acids $1-131$ ) of HFR1 as the site of interaction with the WD40 domain of COP1. This region contains two copies of the putative COP1interacting motif (amino acids 48-83) (Fig. 1B).

To further investigate HFR1-COP1 interaction, we carried out in vitro pull-down assays. Figure 2B shows that HFR1 interacted with the COP1 WD40 domain, but not the COP1 deletion mutant containing only the RING and coiled-coil domains, confirming results obtained with yeast two-hybrid assays. Because the COP1 coiled-coil (designed coil in Fig. 2B) domain is required for dimerization, our results suggest that HFR1 is able to interact with the WD40 domain of COP1 monomer at least in vitro. Surprisingly, the deletion mutant $\operatorname{HFR} 1(\Delta \mathrm{N})$, which lacks the two putative COP1-interacting motifs, was also able to interact with the same WD40 domain, although with reduced affinity. Competition experiments confirmed that the full-length HFR1 has about fourfold greater affinity for the WD40 domain than $\operatorname{HFR} 1(\Delta N)$ (Fig. 2B). These results suggest that, whereas the N-terminal region of HFR 1 is important for binding to the COP1 WD40 domain, other regions of HFR1 also

\section{A}

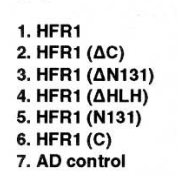

7. AD control

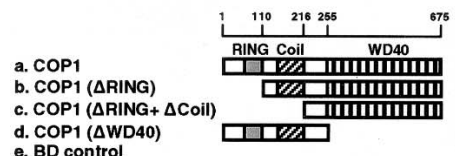

d. $C O P 1(\triangle W D 40)$
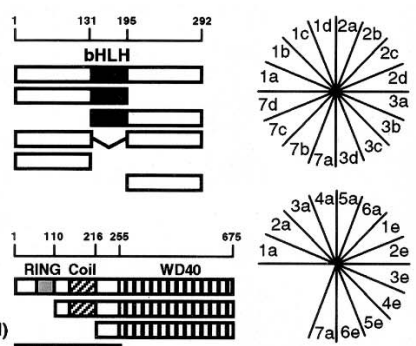

(a)

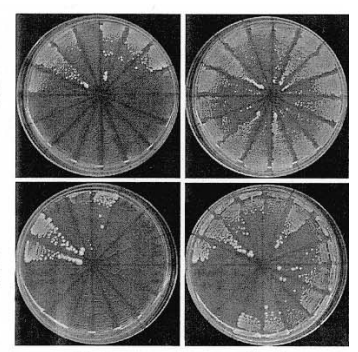

(b)

(c)

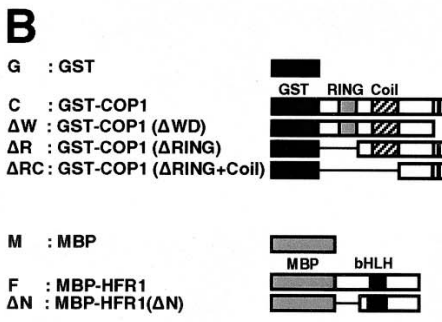

(a)

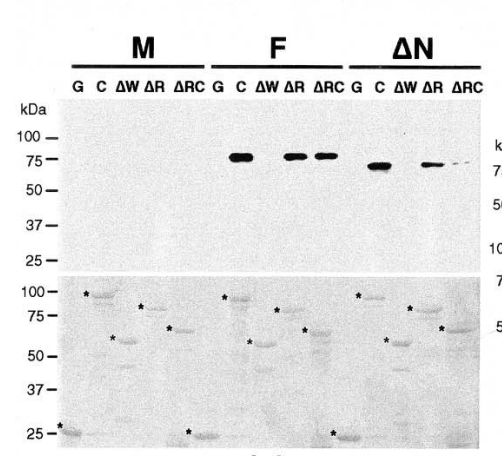

(c)

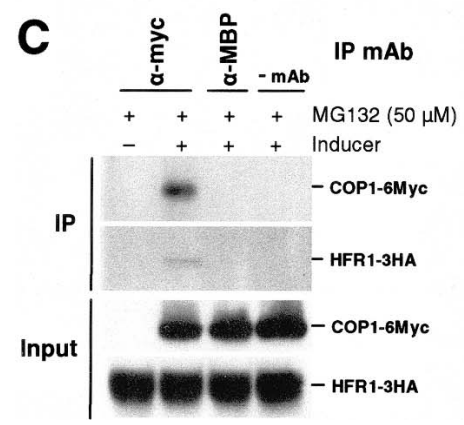

Figure 2. HFR 1 and COP1 interact in vitro and in vivo. (A) Definition of interaction domains between HFR 1 and COP1 by yeast two-hybrid analysis. Different HFR1 cDNA fragments and COP1 cDNA fragments were fused to sequences encoding the Gal4 activation domain (AD) and the Gal4 DNA-binding domain in pGADT7 and pGBT8, respectively. Amino acids for HFR 1 deletion mutant proteins and COP1 mutant proteins are numbered on the scale above the schematic diagrams. The positions of various HFR 1 and COP1 derivatives used are shown in panel $a$. The two vectors were transformed into the yeast strain AH109. Transformants were plated onto minimal medium/-Leu/-Trp/-His (panel b) or /-Leu/-Trp (panel $c$ ) and incubated for $3 \mathrm{~d}$. bHLH in HFR 1 refers to the bHLH domain. $(B)$ In vitro pull-down assays of fulllength HFR1 or HFR1 $(\Delta \mathrm{N})$ with COP1. (Panel $a)$ Schematic diagrams of bait proteins [GST, GST-COP1, GST-COP1 $(\Delta \mathrm{WD})$, GST-COP1( $\Delta \mathrm{RING})$, and GSTCOP1 $(\Delta$ RING + Coil)] and prey proteins [MBP, MBPHFR1, and MBP-HFR $1(\Delta N)]$. Amino acids for bait and prey proteins are identical to those in $A$, except HFR $1(\Delta N)$, which was obtained by deleting amino acid 1-101 from the full-length HFR1. (Panel b) Purified prey proteins were detected by anti-MBP antibody. (Panel c) Two micrograms of prey proteins were pulled-down with the indicated bait proteins $(2 \mu \mathrm{g}$ each) and detected by anti-MBP antibody. Membrane staining after pull-down assay was used to monitor bait proteins loading. Asterisks indicate the bait protein used in each experiment. (Panel $d$ ) Competitive pull-down assay of HFR1 and HFR $1(\Delta N)$. MBP-HFR1 and MBP-HFR $1(\Delta N)$ were mixed in the indicated ratio, pulled down with GST-COP1 or GSTCOP1( $\triangle$ RING + Coil) and detected by anti-MBP-antibody. Amounts of bait proteins ( $1 \mu \mathrm{g}$ each) were fixed in all reactions and $500 \mathrm{ng}$ of prey proteins were used in a 1:1 ratio. Membrane staining after pull-down assay was used to monitor bait proteins loading. Asterisks indicate the bait proteins, $\mathrm{C}$ and $\Delta \mathrm{RC}$. $(C)$ Coimmunoprecipitation of HFR1 with COP1 protein. Extracts of double-transgenic Arabidopsis seedlings (35S-HFR1-3HA and XVE-COP1-6Myc) treated with MG132 $(50 \mu \mathrm{M})$ or MG132 $(50 \mu \mathrm{M})$ plus inducer (10 $\mu M \beta$-estradiol) were immunoprecipitated with antiMyc 9E10 mAb or anti-MBP mAb. Input proteins and the immunoprecipitates were separated on $8 \%$ SDSpolyacrylamide gels, blotted onto membranes, and detected with anti-HA and anti-Myc antibodies. Input refers to the starting protein amount in extracts used for IP reactions. 
contribute to this interaction, albeit with a reduced affinity.

\section{HFR1 interacts with COP1 in vivo}

To see whether HFR1 and COP1 also interacted in vivo, we generated double-transgenic plants expressing 35SHFR 1-3HA and XVE-COP1-6Myc. Note that the latter is an inducible transgene whose expression is dependent on inducer treatment (Zuo et al. 2000). The extreme instability of HFR1 in vivo promoted us to block its degradation in transgenic plants by the proteasomal inhibitor, MG132 (see latter sections). Figure 2C shows that immunoprecipitation of COP1-6Myc, which was expressed only upon inducer treatment, also pulled down HFR1. Although the recovery of HFR1-3HA was low, the interaction was clearly specific and dependent on COP16Myc, as HFR1-3HA was not detected in the noninduced sample. Neither COP1-6Myc nor HFR1-3HA was detected in the absence of the monoclonal antibody or when anti-MBP antibody was used as a negative control. The low HFR1-3HA levels in the coimmunoprecipitate could be attributed to at least two factors: (1) Only a minor proportion of the total cellular HFR1-3HA was associated with COP1-6Myc, and (2) the interaction between COP1-6Myc and HFR1-3HA may be weak, and did not survive the stringent wash conditions used in our experiments.

\section{In vitro ubiquitination}

The interaction of HFR1 with COP1 provides preliminary evidence that the former may be a substrate of the latter. We purified HFR1-3HA recombinant protein from Escherichia coli extracts and used it as a substrate in an in vitro ubiquitination reaction. Figure 3 shows that HFR1 was polyubiquitinated by COP1 E3 ligase in a reaction dependent on E1 and E2 activities. We used SINAT5, an E3 ligase that modifies NAC1 (Xie et al. 2002), as a negative control. The inability of SINAT5 to modify HFR 1 indicates specificity of the reaction. Similar results were obtained using $\operatorname{HFR} 1(\Delta N)$, although the ubiquitination reaction was not as efficient and produced mainly mono- and di-ubiquitinated products (Fig. 3, cf. A and B). Nonetheless, polyubiquitinated $\operatorname{HFR} 1(\Delta N)$ could be detected upon longer exposure (data not shown). This presumably resulted from the weak interaction between $\operatorname{HFR} 1(\Delta N)$ and COP1.

\section{Differential stability of HFR1 and $\operatorname{HFR} 1(\Delta N)$ in transgenic plants}

To examine the stability of HFR 1 and $\operatorname{HFR} 1(\Delta N)$ in vivo, we constructed cDNAs encoding the two HFR 1 proteins tagged with $3 \mathrm{HA}$ at the $\mathrm{C}$ terminus. When expressed from a CaMV35S promoter, full-length HFR1, as well as the deletion mutant $\operatorname{HFR} 1(\Delta \mathrm{N})$, were able to complement the $h$ fr1-201 mutation (Soh et al. 2000) with respect to hypocotyl length under blue (data not shown) and FR light. This result indicates that the biological activity of HFR 1 and its deletion mutant was not compromised by the attachment of the $3 \mathrm{HA}$. Here, we focus on FR-induced photoresponses. At low fluences, transgenic lines expressing the $\operatorname{HFR} 1(\Delta \mathrm{N})$ mutant were hypersensitive to (FR) light with respect to hypocotyl elongation as well as cotyledon expansion (Fig. 4A, panel a). In addition, these lines also displayed constitutive photomorphogenesis in the dark with unfolded and expanded cotyledons as well as shorter hypocotyls (Fig. 4A, panel b). These results confirm previous observations of Yang et al. (2003) who used an HFR1 deletion mutant lacking the N-terminal 105 amino acids (HFR1- $\Delta$ N105). Western blot analysis revealed a much higher expression level of $\operatorname{HFR} 1(\Delta N)$ than HFR1 (Fig. 4A, panel c).

Further work was performed with wild-type transgenic lines expressing 35S-HFR1-3HA, 35S-HFR1-6Myc, 35SHFR $1(\Delta N)-3 H A$, and $35 S-H F R 1(\Delta N)-6 M y c$ (Fig. 4B). We screened five independent lines each of 35S-HFR1-3HA and 35S-HFR1-6Myc. All showed a similar phenotype with a slight hypersensitivity to low-fluence FR, as compared with wild-type plants. Figure 4B shows hypocotyl lengths and cotyledon phenotypes of two representative lines for each construct. Similar results were obtained with lines expressing $\operatorname{HFR} 1(\Delta N)$, except that some lines displayed greater hypersensitivity to FR as well as constitutive photomorphogenesis in darkness. Western blot analysis showed that the expression level of full-length HFR1 protein was either very low (lines A1 and A3; tagged with 3HA) or not detectable (lines B1 and B3; tagged with $6 \mathrm{Myc})$ compared with that of $\operatorname{HFR} 1(\Delta N)$ in either darkness or FR light (Fig. 4C). Among the four transgenic lines $(\mathrm{C} 1, \mathrm{D} 1-3)$ expressing $\operatorname{HFR} 1(\Delta \mathrm{N})$, the phenotypic severity in darkness or in FR light correlated with the protein expression level.

Northern blot analysis of lines A1 (35S-HFR1-3HA) and $\mathrm{C} 1[35 S-H F R 1(\Delta N)-3 H A]$ showed that the HFR1$3 H A$ transcript level was about twofold lower than that

\footnotetext{
Figure 3. In vitro ubiquitination of HFR1 or $\operatorname{HFR} 1(\Delta N)$ by COP1. Epitope-tagged recombinant HFR1, HFR $1(\Delta N), C O P 1$, and SINAT5 were expressed in $E$. coli, and in vitro ubiquitination assays were performed as described in Materials and Methods. COP1-mediated ubiquitination of HFR1 $(A)$ and $\operatorname{HFR} 1(\Delta N)(B)$ was confirmed by Western blot analyses. (MBP) Maltose-binding protein; (MBP-COP1 and MBP-SINAT5) COP1 and SINAT5 tagged with MBP.
}

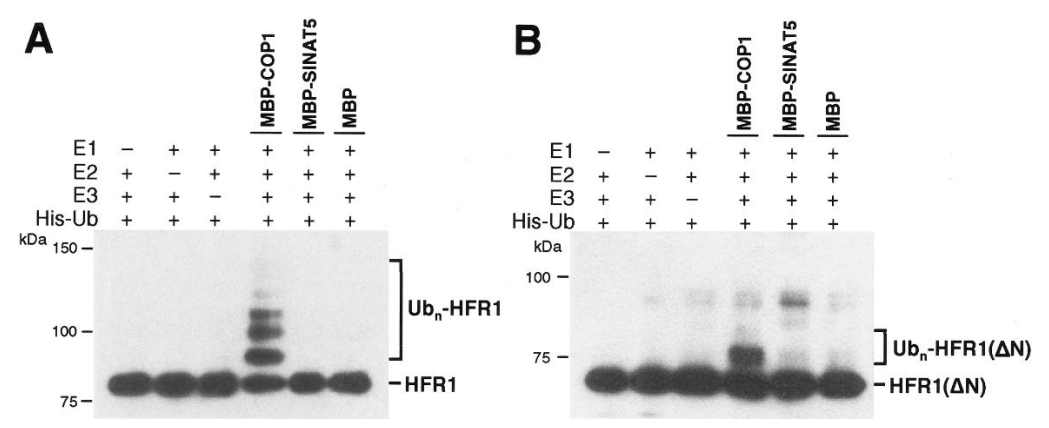




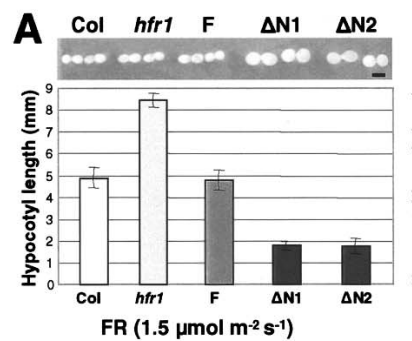

(a)

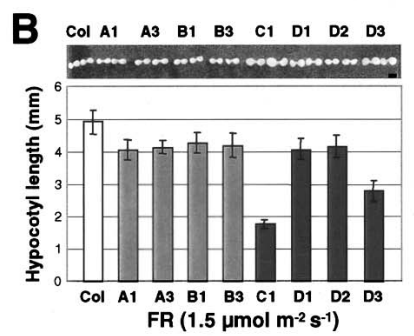

(a)

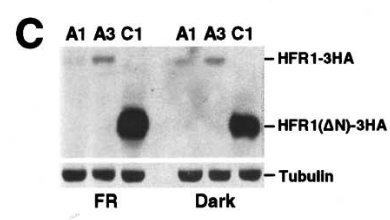

B1 B3 D1 D2 D3 B1 B3 D1 D2 D3

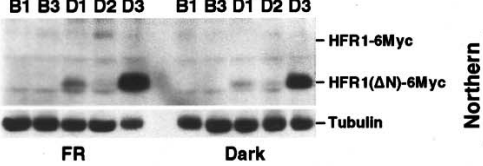

FR

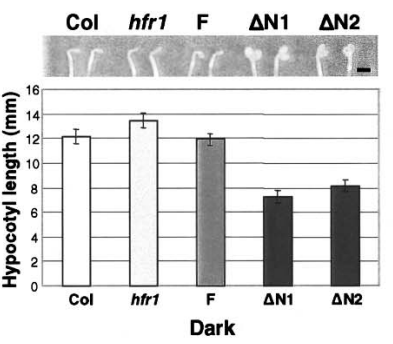

(b)

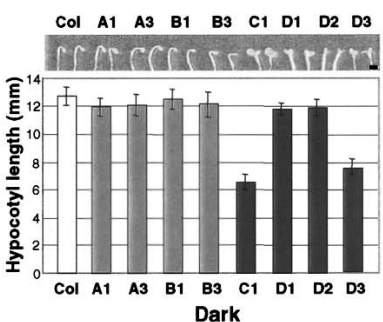

(b)

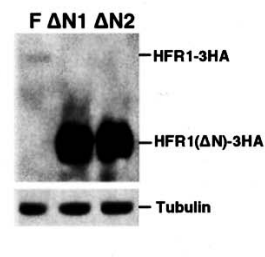

(c)
E

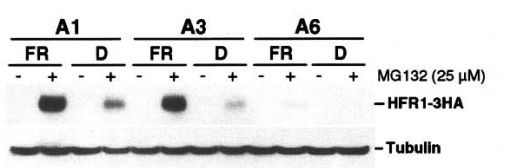

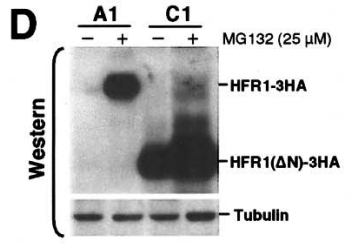
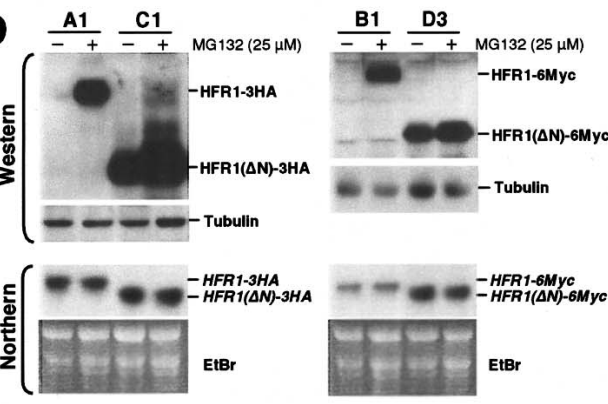

Figure 4. Phenotypes of transgenic Arabidopsis seedlings expressing HFR1 or $\operatorname{HFR} 1(\Delta N)$. Seedlings were grown for $4 \mathrm{~d}$ under FR light $\left(1.5 \mu \mathrm{mol} \mathrm{m} \mathrm{m}^{-2} \mathrm{~s}^{-1}\right)$ or in darkness on medium that was not supplemented with sucrose. Data were presented as average hypocotyl length \pm standard deviations (SD; $n=15)$. (A) Complementation of hfr1 mutant by full-length HFR1$3 \mathrm{HA}$ or $\operatorname{HFR} 1(\Delta \mathrm{N})-3 \mathrm{HA}$ overexpression under FR light (panel $a$ ) or in darkness (panel $b$ ). Cotyledons from representative seedlings are shown above the histograms. Bar, $1 \mathrm{~mm}$. (Panel c) Western blot analysis of seedlings treated with FR light (1.5 umol m $\mathrm{m}^{-2} \mathrm{~s}^{-1}$ ) for $4 \mathrm{~d}$. (Col) Columbia wild type; (hfr1), hfr1-201 mutant (Soh et al. 2000); (F) Full-length HFR1-overexpressing transgenic line in $h$ fr $1-201 ;(\Delta \mathrm{N} 1$ and $\Delta \mathrm{N} 2)$ two independent $\operatorname{HFR} 1(\Delta \mathrm{N})$-overexpressing lines in $h f r 1-201$. (B) Hypersensitivity of $\operatorname{HFR} 1(\Delta \mathrm{N})$-overexpressing transgenic seedlings to FR light (panel $a$ ) and constitutive photomorphogenesis of the seedlings in darkness (panel b). (A1 and A3) HFR1-3HA-overexpressing transgenic lines; (B1 and B3) HFR1-6Myc-overexpressing transgenic lines; (C1) HFR $1(\Delta N)$ 3HA-overexpressing transgenic line; (D1, D2, and D3) HFR1 $(\Delta \mathrm{N})$-6Myc-overexpressing transgenic lines. Cotyledons of representative seedlings are shown above the histograms. Bar, $1 \mathrm{~mm}$. (C) Western blot analysis of selected lines after the treatments described for $B$. Tubulin levels were used as loading controls. $(D)$ Western and Northern blot analyses of seedlings treated with MG132 after FR treatments described in $B$. After treatment with $25 \mu \mathrm{M}$ MG132 or mock-treatment, FR-irradiated seedlings were incubated for a further $12 \mathrm{~h}$ under continuous FR light $\left(1.5 \mu \mathrm{mol} \mathrm{\textrm {m } ^ { - 2 }}\right.$ $\left.\mathrm{s}^{-1}\right)$. 3HA and 6Myc-specific cDNA probes were used to detect transgene-specific transcripts. Each lane contained $5 \mu \mathrm{g}$ RNA (E) Western blot analysis of transgenic Arabidopsis seedlings overexpressing HFR1-3HA in darkness or under FR irradiation with or without MG132. After treatment with $25 \mu \mathrm{M}$ MG132 or mock treatment, FR-irradiated or dark-grown seedlings were incubated for a further $12 \mathrm{~h}$ under continuous FR light $\left(1.5 \mu \mathrm{mol} \mathrm{m} \mathrm{m}^{-2} \mathrm{~s}^{-1}\right)$ or in darkness. Tubulin levels were used as loading controls.

of HFR1( $\triangle N)-3 H A$. Nevertheless, the basal HFR1-3HA protein level was very low and at least 25 -fold lower than that of HFR $1(\Delta \mathrm{N})-3 \mathrm{HA}$. The HFR1-3HA level could be considerably enhanced (25- to 50 -fold) by the addition of MG132, a proteasome inhibitor. In contrast, only a moderate increase (two- to threefold) was seen with HFR1 $(\Delta N)-3 H A$ under the same conditions (Fig. 4D). We conclude that HFR1-3HA is extremely unstable as compared with the deletion mutant HFR $1(\Delta \mathrm{N})-3 \mathrm{HA}$. Similar differential stability between HFR1-6Myc and HFR $1(\Delta N)-6$ Myc was also observed in lines B1 and D3 (Fig. 4D).

These results are consistent with the observation of a higher polyubiquitination efficiency of HFR1 than the deletion mutant in vitro.

We used three independent lines of HFR1-3HA-overexpressing plants to further compare the effects of
MG132 on HFR1 levels in darkness and FR light (Fig. 4E). Under both conditions, HFR-3HA levels were either very low or undetectable, but can be significantly elevated by MG132 treatment. However, in the presence of the proteasome inhibitor, HFR1-3HA levels in all three lines were severalfold higher in FR compared with darkness, suggesting a higher steady HFR1 levels in FR light.

\section{HFR1 levels are increased and decreased by inducible expression of DN-COP1 and wild-type COP1, respectively}

The instability of HFR 1 in vivo raises the question of the identity of its cognate E3 ligase. Because of the observed in vitro and in vivo interactions between HFR1 and COP1, we hypothesized that HFR 1 is targeted by COP1 
for degradation in vivo. To test this hypothesis, we analyzed double-transgenic plants expressing 35S-HFR1$3 H A$ and the estradiol inducible XVE-COP1-6Myc. More than 10 independent transgenic lines with similar phenotypes were obtained and three lines (\#1-3) were analyzed in some detail.

Because of its extreme instability, HFR1 was almost undetectable in all transgenic lines expressing 35SHFR1-3HA (data not shown; see also Fig. 4). However, increased accumulation of this protein could be obtained by treating transgenic plants with low concentration of MG132 (10 $\mu \mathrm{M})$, which partially blocks proteasomal degradation. Under this condition, induced expression of COP1-6Myc clearly resulted in a decrease in HFR1-3HA levels (Fig. 5A). Northern blot analysis showed that expression of the HFR1-3HA transcripts was comparable in samples with or without MG132 (Fig. 5A).

If COP1 acts as an E3 ligase for HFR1 in vivo, HFR1 protein levels should increase when COP1 E3 activity is compromised. We have previously shown that mutations in critical amino acid residues of COP1 block its E3 liagse activity, and in addition, COP1 RING mutant can function in a dominant-negative manner by forming inactive heterodimers with endogenous COP1 (Seo et al. 2003, 2004). We analyzed three independent transgenic lines expressing 35S-HFR1-3HA and XVE-COP1(DN)$6 M y c$. In contrast to induced expression of transgenic wild-type COP1 (Fig. 5A), induced expression of the dominant-negative mutant (COP1-DN) resulted in an increase, rather than a decrease, of HFR1 levels. Because no significant changes in HFR1 transcript levels were observed (Fig. 5B), our results are consistent with the notion that HFR 1 is targeted by COP1 for destruction by a post-translational event.

To further confirm that COP1 triggers post-translational destruction of HFR1, we determined the time course of HFR1 levels after new protein synthesis in transgenic seedlings was inhibited by cycloheximide. Figure 5C shows that HFR1 protein had a half life of only $\sim 2.0$ and $0.5 \mathrm{~h}$ in white and FR light, respectively. In both cases, induced expression of wild-type COP1 clearly accelerated HFR1 destruction rate.

\section{Discussion}

\section{HFR1 interacts with COP1}

Among the three transcription factors that are known to mediate phyA signaling, HY5 and LAF1 have been shown to colocalize with COP1 in nuclear bodies. The
Figure 5. HFR 1 is targeted by COP1 for degradation by $26 \mathrm{~S}$ proteasomes. $(A) \mathrm{HFR} 1$ protein levels are decreased by induced COP1 expression. (Panel $a$ ) Three independent transgenic lines containing 35S-HFR1-3HA and $X V E-C O P 1-6 M y c$ were treated with MG132 (10 $\mu \mathrm{M})$ with and without induction of COP1-6Myc expression by inducer (10 $\mu \mathrm{M} \beta$-estradiol) for $16 \mathrm{~h}$. HFR 1 and COP1 expression levels were detected by anti-HA and antiMyc antibodies, respectively. Tubulin expression was used to normalize loading. (Panel $b$ ) Northern blot analysis of transgenic plants containing 35S-HFR1$3 H A$ and $X V E-C O P 1-6 M y c$. Total RNAs were isolated from transgenic plants treated as in panel $a$. Transgenic HFR 1 transcript levels were detected using a 3HA-specific probe. Ethidium bromide-staining (EtBr) of total RNAs ( $5 \mu \mathrm{g} / \mathrm{lane}$ ) was used to monitor RNA loading. $(B)$ HFR 1 protein levels are increased by expression of dominant-negative (DN) COP1 mutant. (Panel a) Three independent transgenic lines containing 35S-HFR1$3 H A$ and $X V E-C O P 1(D N)-6 M y c$ were treated with and without inducer (10 $\mu \mathrm{M} \beta$-estradiol) for $16 \mathrm{~h}$. Antibodies for detection of HFR1-3HA, COP1(DN)-6Myc, and loading control were identical to those of $A$. (Panel $b$ ) Northern blot analysis of transgenic plants containing 35S-HFR1-3HA and XVE-COP1(DN)-6Myc. Total RNAs were isolated from transgenic plants treated as in panel $a$. All conditions were identical to those of $A .(C)$ HFR1-3HA expression is regulated post-translationally by COP1-6Myc. Ten-day-old double-transgenic Arabidopsis seedlings (35S-HFR1-3HA and XVE-COP1$6 \mathrm{Myc})$ were incubated in liquid MS medium with MG132 $(50 \mu \mathrm{M})$ or MG132 $(50 \mu \mathrm{M})$ plus $\beta$-estradiol $(10 \mu \mathrm{M})$ for $16 \mathrm{~h}$, washed, and then transferred to MS medium with $1 \mathrm{mM}$ cycloheximide (CHX). Treated seedlings were exposed to white light or FR light. Proteins were extracted at the indicated times and analyzed by Western blotting using anti-HA or anti-Myc (Santa Cruz Biotechnology) antibodies. Tubulin levels as detected by anti-tubulin antibody were used as loading controls. Expression levels of HFR1 and tubulin were calculated using the program of Image Gauge V3.12 (Fuii) and the values were normalized to 0 time in the (-) inducer sample of both panels.

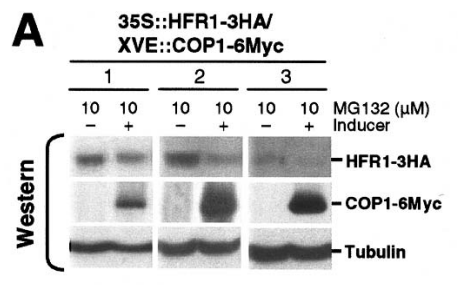

(a)

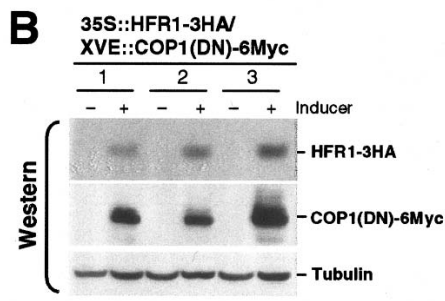

(a)

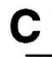

C
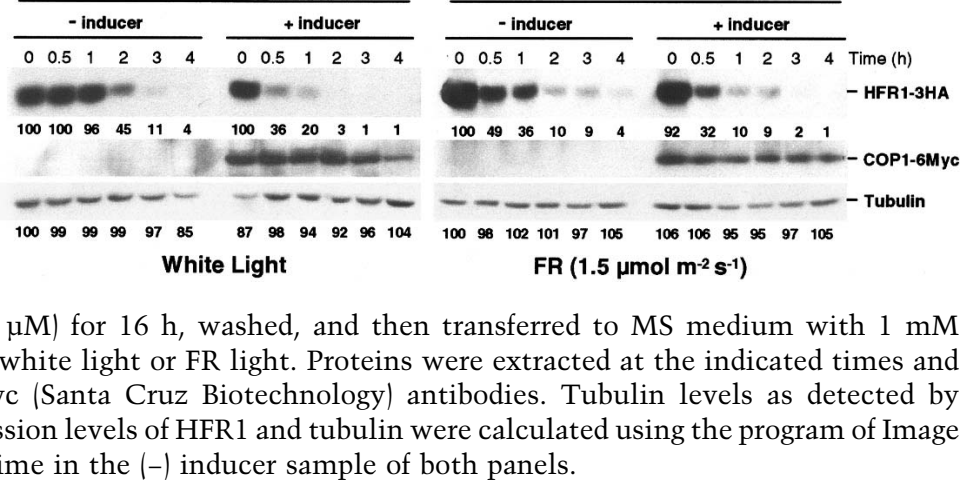
bZIP protein HY5 (Holm et al. 2001b) interacts with the COP1 WD40 domain, while the myb transcription factor LAF1 (Ballesteros et al. 2001) binds to the RING motif (Seo et al. 2003). Here, we show that HFR1, a basic helixloop-helix (bHLH) protein (Fairchild et al. 2000; Fankhauser and Chory 2000; Soh et al. 2000) also localizes to COP1/HY5/LAF1 nuclear bodies. Although not a bZIP protein, HFR1 also interacts with the COP1 WD40 domain (Fig. 2). It is therefore not surprising that this factor contains the putative COP1-interacting motif first identified in HY5 (Holm et al. 2001b) and later found in two other COP1-interacting proteins (HYH and c-Jun) as well (Holm et al. 2001a,b; Bianchi et al. 2003). The two putative COP1-interacting motifs of HFR1 are located within the N-terminal 86 amino acids (Fig. 1B). Nevertheless, deletion of the first 101 amino acids only reduced, but did not completely block binding to COP1 (Fig. 2). This result suggests that sequences mediating low-affinity binding to COP1 are present in the $\operatorname{HFR} 1(\Delta \mathrm{N})$ mutant.

COP1 E3 ligase has been previously shown to ubiquitinate LAF1 (Seo et al. 2003), HY5 (Saijo et al. 2003), and phyA (Seo et al. 2004). The demonstration that HFR1 can be ubiquitinated in vitro brings the total number of identified COP1 substrates to four. Consistent with the lower affinity binding to COP1, the deletion mutant $\operatorname{HFR} 1(\Delta N)$ was ubiquitinated with a lower efficiency compared with full-length HFR1 (Fig. 3).

\section{HFR1 is very unstable in vivo}

The association of HFR1 with the E3 ligase COP1 suggests that the transcription factor may be unstable in vivo. To investigate this issue, we first verified that HAtagged versions of full-length HFR1, as well as $\operatorname{HFR} 1(\Delta \mathrm{N})$, were able to complement the $h$ fr1-201 mutation (Fig. 4A). In wild type, as well as the $h f r 1-201 \mathrm{mu}-$ tant, the steady-state level of HFR1 was at least 25-fold lower than that of $\operatorname{HFR} 1(\Delta N)$ (Fig. 4A,C). As shown by Northern blot analysis, this difference in the steady-state level between the full-length HFR1 and the deletion mutant cannot be explained by a difference in transcript levels (Fig. 4D). Indeed, inhibition of $26 \mathrm{~S}$ proteasome activity by MG132 led to a massive accumulation of HFR1 in wild-type plants (Fig. 4D), as well as in hfr1-201 (data not shown). In contrast, only a two- to threefold increase in $\operatorname{HFR} 1(\Delta N)$ levels was seen under the same conditions. These results indicate that HFR1 level is dependent on 26S proteasome activity, and that a major HFR1 instability determinant resides in the $\mathrm{N}$-terminal 101 amino acids absent in $\operatorname{HFR} 1(\Delta N)$. Because this $\mathrm{N}$-terminal region contains the two COP1-interacting motifs, it is reasonable to assume that the association of HFR1 with COP1 leads to HFR1 polyubiquitination and presumably subsequent degradation. As predicted, $\operatorname{HFR} 1(\Delta N)$, which lacks this region, is considerably more stable than fulllength HFR1.

Yang et al. (2003) expressed 35S-HFR1 and 35S-HFR1$\Delta N 105$ in wild type as well as the $h$ fr1-201 mutant. In spite of comparable transgene transcript levels, they found that HFR1 overexpression did not produce any significant photoresponses, while HFR1- $\Delta$ N105 overexpression elicited constitutive photomorphogenesis in darkness and hypersensitivity to FR. Similar transgenic phenotypes were obtained in our experiments. The extreme instability of HFR1 may explain the absence of a FRhypersensitive phenotype, even in transgenic plants overexpressing HFR1, because HFR1 protein accumulation constrains the photoresponses. In contrast, expression of $\operatorname{HFR} 1(\Delta N)$ in wild type produced higher protein levels, leading to constitutive photomorphogenesis in the dark and hypersensitivity to low-fluence FR light. The activity of $\operatorname{HFR} 1(\Delta N)$ in dark grown seedlings suggests that this factor is active in darkness. Analysis of hfr1-201/cop1-6 double mutants led to a similar conclusion (Kim et al. 2002).

We found that HFR1-3HA is very unstable in FR light as well as in darkness; however, massive accumulation of this protein can be detected when proteasomal degradation is blocked (Fig. 4E). Under this condition, the steady HFR1-3HA level is higher in FR light compared with darkness. This observation of a greater abundance of HFR1-3HA in FR is consistent with its role in phyA signaling.

\section{COP1 is an E3 ligase for HFR1 in vivo}

The interaction of HFR 1 and COP1 in vitro, the ubiquitination of the former by the latter in vitro, the colocalization of the two proteins in nuclear bodies, and their association in vivo as revealed by coimmunoprecipitation all suggest that HFR1 is also modified by COP1 in vivo. Evidence for the in vivo relationship between the two proteins was obtained by analysis of HFR1 levels in double-transgenic plants expressing 35S-HFR1-3HA and XVE-COP1-6Myc or 35S-HFR1-3HA and XVE$C O P 1(D N)-6 M y c$. Owing to the very low HFR1 levels in these plants, the effects of wild-type COP1 could only be revealed when HFR1 degradation was partially blocked by MG132 (Fig. 5). At $10 \mu M$ MG132, induced expression of COP1 clearly reduced HFR1 abundance in three independent transgenic lines. In contrast, induced expression of a dominant-negative mutant of COP1, which compromises endogenous COP1 E3 activity, stabilized HFR1. Finally, using protein synthesis inhibitor, we demonstrated that COP1-mediated HFR1 degradation is a posttranslational event.

These results provide evidence that COP1 serves as an E3 ligase to ubiquitinate HFR1 in vivo and target the latter for rapid destruction by $26 \mathrm{~S}$ proteasomes. However, we should emphasize that ubiquitination of HFR1 may be mediated by more than one E3 ligase, since there are several examples of differential utilization of E3 ligases to modify one substrate, depending on the physiological context. The identification of HFR1 as a substrate of COP1 in vivo explains the previous observation that HFR 1 is required for a subset of photoresponses triggered by cop1 mutations (Kim et al. 2002). 


\section{Concluding remarks}

Based on cotyledon morphology and hypocotyl lengths under FR light, hy5, laf1, and hfr1 mutants all have less severe phenotypes than those of phyA null mutants. One interpretation of this observation is that there are several phyA-activated pathways, each regulated by different signaling components. Consistent with this interpretation, double mutants of hy5-1/hfr1-201 display a more severe phenotype than do either of the single mutants (Kim et al. 2002). Our results on the gain-of-function of $\operatorname{HFR} 1(\Delta \mathrm{N})$ and those of Yang et al. (2003) on HFR1$\Delta \mathrm{N} 105$ further support this view. Together, these results show that two closely similar N-terminal deletion mutants of HFR1 can phenocopy cop1 mutants in the dark and FR light, although the phenotype is not as severe. As LAF1 and HY5 are also substrates of COP1, it is reasonable to assume that, whereas these two signaling components are not degraded in cop1 mutants, they continue to be degraded in transgenic plants overexpressing $\operatorname{HFR} 1(\Delta \mathrm{N})$ or HFR1- $\Delta$ N105. The different stabilities of HY5 and LAF1 in cop 1 mutants and HFR $1(\Delta \mathrm{N})$ transgenic plants may account, at least in part, for the phenotypic difference.

Together with previous reports (Osterlund et al. 2000; Saijo et al. 2003; Seo et al. 2003), our work here emphasizes the importance of COP1 E3 ligase in regulating protein levels of phyA signaling factors (HFR1, HY5, LAF1) that interact with COP1. At present, it is not known whether the abundance of such factors is regulated by changes in intracellular translocation of COP1 and/or changes in its E3 activity by protein modification or association with regulatory proteins. A different mechanism may regulate factors that do not interact with COP1, e.g., PAT1 (Bolle et al. 2000). A major challenge in the future is to understand how different pathways are integrated to bring about a full phyA response.

\section{Materials and methods}

\section{Subcellular localization experiment}

The YFP coding sequence was fused in-frame to the $3^{\prime}$ ends of full-length $H F R 1$ cDNA and cDNA encoding the HFR $1(\Delta \mathrm{N})$ deletion mutant to generate HFR1-YFP and HFR1 $(\Delta N)-Y F P$, respectively. Both fusion genes were transcribed from a $35 \mathrm{~S}$ promoter. The CFP-COP1 construct was described elsewhere (Seo et al. 2003). Two micrograms of each plasmid was bombarded into the epidermis of the inner surface of onion scales using a particle gun-mediated system (Bio-Rad). Bombarded tissues were incubated in darkness for $12 \mathrm{~h}$ before visualization of transient expression using a confocal laser-scanning microscope (Carl Zeiss LSM510) with a standard filter set.

\section{Yeast two-hybrid assays}

Yeast two-hybrid assays were performed using the Matchmaker GAL4-based two- hybrid system as recommended (Clontech). Full-length HFR 1 and COP1 cDNA fragments were cloned into pGADT7 and pGBT8 (Clontech) to generate the constructs $A D$ HFR 1 and $B D-C O P 1$, respectively. HFR 1 and COP1 deletion mutants were generated by PCR and cloned into pGADT7 and
pGBT8, respectively. All constructs were transformed into yeast strain AH109 by the lithium acetate method and yeast cells were grown on minimal medium/-Leu/-Trp according to the manufacturer's instructions (Clontech). Transformed colonies were plated onto minimal medium/-Leu/-Trp/-His to test for possible interactions between HFR1 and COP1.

\section{Preparation of recombinant proteins}

cDNAs encoding full-length and deletion mutants of Arabidopsis COP1 cDNA were amplified by PCR and inserted into pGEX-4T-1 (Amersham Biosciences) to generate GST-COP1 encoding full-length of COP1, GST-COP1 $(\Delta \mathrm{WD})$ encoding amino acids 1-255 of COP1, GST-COP1 ( $\triangle$ RING) encoding amino acids 110-675 of COP1, and GST-COP1 ( $\triangle$ RING + Coil) encoding amino acids 216-675 of COP1. cDNAs encoding full-length HFR 1 and its deletion mutant $\operatorname{HFR} 1(\Delta N)$ were amplified by PCR and cloned into pMal-c2 (New England Biolabs) to generate MBP-HFR1 encoding full-length HFR 1 and MBP-HFR1 $(\Delta N)$ encoding amino acids 102-292 of HFR1. To enable detection of fusion proteins in in vitro ubiquitination assays, a DNA fragment encoding three copies of hemagglutinin (3HA; LopezMolina et al. 2003) was inserted into pMal-c2 (New England Biolabs) to generate pMal-c2-3HA. cDNAs encoding full-length HFR 1 and its deletion mutant $\operatorname{HFR} 1(\Delta N)$ were cloned into pMal-c2-3HA to generate MBP-HFR1-3HA and MBPHFR1 $(\Delta \mathrm{N})-3 \mathrm{HA}$, respectively. Plasmid encoding MBP-SINAT5 was described previously (Seo et al. 2003; Xie et al. 2002).

All constructs were transformed into $E$. coli BL21 cells that were treated with isopropyl- $\beta-D$-thiogalactoside to induce fusion protein expression. Treated cells were broken by a French pressure cell press (SIM AMINCO) in purification buffer (20 mM Tris-Hcl at $\mathrm{pH} 7.4 ; 200 \mathrm{mM} \mathrm{NaCl} ; 1$ mM EDTA; $1 \%$ Triton $\mathrm{X}-100 ; 2 \mathrm{mM}$ phenylmethylsulphonyl fluoride) containing a proteinase inhibitor cocktail (Roche). Proteins were bound to amylose resin (New England Biolabs) for MBP-fused proteins and glutathione Sepharose 4B (Amersham Biosciences) for GSTfused proteins, washed with the purification buffer, and eluted from the column using purification buffer containing $10 \mathrm{mM}$ maltose (for MBP-fused proteins) or $10 \mathrm{mM}$ glutathione (for GST-fused proteins).

In vitro pull down, in vitro ubiquitination assays, and in vivo coimmunoprecipitation

For in vitro pull-down assays (Seo et al. 2004), $2 \mu \mathrm{g}$ of bait protein (full-length COP1 or its deletion mutants) and $2 \mu \mathrm{g}$ of prey protein (full-length HFR1 or its deletion mutant) were incubated at $25^{\circ} \mathrm{C}$ for $2 \mathrm{~h}$ in binding buffer $(50 \mathrm{mM}$ Tris-Hcl at $\mathrm{pH}$ 7.5; $100 \mathrm{mM} \mathrm{NaCl} ; 0.6 \%$ Trition X-100; $0.5 \mathrm{mM} \beta$-mercaptoethanol) and further incubated with glutathione sepharose $4 \mathrm{~B}$ for $2 \mathrm{~h}$. After washing with buffer $(50 \mathrm{mM}$ Tris-Hcl at $\mathrm{pH} 7.5$; $100 \mathrm{mM} \mathrm{NaCl} ; 0.6 \%$ Trition X-100), pulled-down proteins were separated on $10 \%$ SDS-polyacrylamide gels and detected by Western blotting using anti-MBP antibody (Santa Cruz Biotechnology). For competitive pull-down assays, $1 \mu \mathrm{g}$ of bait proteins and $500 \mathrm{ng}$ of prey proteins were used.

For in vitro ubiquitination assays (Seo et al. 2003), each reaction mixture $(30 \mu \mathrm{L})$ contained $\sim 100$ ng protein substrate [MBPHFR1-3HA or MBP-HFR1( $\Delta \mathrm{N})-3 \mathrm{HA}], 20$ ng rabbit E1 (Boston Biochem), $20 \mathrm{ng}$ human E2 UbcH5b (Boston Biochem), $10 \mu \mathrm{g}$ His $_{6}$-ubiquitin (Sigma) and $200 \mathrm{ng}$ E3 (COP1 or SINAT5). Reactions were carried out at $30^{\circ} \mathrm{C}$ for $2 \mathrm{~h}$. Ten microliters of the reaction mixtures were separated on $8 \%$ SDS-polyacrylamide gel, and ubiquitinated MBP-HFR1-3HA or MBP-HFR1 $(\Delta \mathrm{N})$-3HA 
was detected by Western blotting with anti-HA (Santa Cruz biotechnology) antibody.

For in vivo coimmunoprecipitation, 10-d-old double-transgenic Arabidopsis seedlings (35S-HFR1-3HA and XVE-COP1$6 \mathrm{Myc})$ treated with MG132 $(50 \mu \mathrm{M})$ or MG132 $(50 \mu \mathrm{M})$ plus $\beta$-estradiol $(10 \mu \mathrm{M})$ for $16 \mathrm{~h}$ were ground in liquid- $\mathrm{N}_{2}$. The powder was resuspended by homogenization at $4^{\circ} \mathrm{C}$ in buffer A (50 $\mathrm{mM}$ Tris-Hcl at $\mathrm{pH} 7.5 ; 100 \mathrm{mM} \mathrm{NaCl} ; 0.2 \%$ Triton X-100; 1 $\mathrm{mM}$ DTT) containing proteinase inhibitor cocktail (Roche). After centrifugation, the supernatants were used for immunoprecipitation reactions. Approximately $1 \mathrm{mg}$ of total protein was precleared by addition of $20 \mu \mathrm{L}$ of protein A agarose beads (Santa Cruz Biotechnology), incubation for $1 \mathrm{~h}$ at $4^{\circ} \mathrm{C}$, and centrifugation at $10,000 \times \mathrm{g}$ for $1 \mathrm{~min}$. Five micrograms of anti-Myc monoclonal 9E10 (Abcam) antibody or anti-MBP monoclonal (Santa Cruz biotechnology) antibody were added to the precleared extract, and incubated for $1 \mathrm{~h}$ at $4^{\circ} \mathrm{C}$. Twenty microliters of protein $\mathrm{A}$ agarose beads were added to the mixture, and further incubated for $1 \mathrm{~h}$ at $4^{\circ} \mathrm{C}$. After centrifugation, the beads were washed five times with extraction buffer and proteins were eluted by heating at $95^{\circ} \mathrm{C}$ for $10 \mathrm{~min}$ in $100 \mu \mathrm{L}$ of $2 \times$ sample buffer. Eluted proteins were analyzed by Western blotting using anti-HA and anti-Myc (Santa Cruz Biotechnology) antibodies.

Plant material, transformation vectors, and production of transgenic plants

The wild-type Arabidopsis thaliana Columbia ecotype and hfr1-201 (in the Columbia background; Soh et al. 2000) were used in this study. Seed sterilization and growth conditions were as described (Xie et al. 2000). Conditions for FR irradiation were described previously (Bolle et al. 2000). Plant transformation constructs were based on the plasmids pBA002 (Kost et al. 1998) and pER8 (Zuo et al. 2000). For detection of transgene expression, a DNA fragment encoding three copies of HA was inserted into pBA002 to generate pBA002-3HA. cDNAs encoding full-length HFR 1 and its deletion mutant $\operatorname{HFR} 1(\Delta N)$ were cloned into pBA002-3HA to place expression of these 3HA fusions under the control of the CaMV $35 \mathrm{~S}$ promoter. $\mathrm{XVE}: \mathrm{COP1-6Myc}$ was constructed for $\beta$-estradiol inducible expression in plants by cloning into pER 8 (Seo et al. 2003). Plants were transformed by the Agrobacterium tumefaciens vacuuminfiltration method (Clough and Bent 1998).

\section{Protein extraction and Western blotting}

Approximately $100 \mathrm{mg}$ of whole Arabidopsis seedlings was frozen in liquid- $\mathrm{N}_{2}$ and ground to a fine powder using a mortar and a pestle. The powder was resuspended by homogenization at $4^{\circ} \mathrm{C}$ in buffer $(125 \mathrm{mM}$ Tris- $\mathrm{Hcl}$ at $\mathrm{pH} 8.8 ; 1 \%$ SDS; $10 \%$ glycerol; $50 \mathrm{mM} \mathrm{Na}_{2} \mathrm{~S}_{2} \mathrm{O}_{5} ; 2 \mathrm{mM}$ PMSF) containing proteinase inhibitor cocktail (Roche). After homogenization, the mixture was clarified by centrifugation and protein concentration was determined using the Dc protein assay (Bio-Rad). Protein extracts $(10 \mu \mathrm{g})$ were separated on $10 \%$ SDS-polyacrylamide gels and transferred to a polyvinylidene difluoride (PVDF) membranes (Immobilon-P, Millipore) using an electro transfer apparatus (Bio-Rad). The membranes were incubated with anti-tubulin (Sigma) or anti-HA or anti-Myc (Santa Cruz Biotechnology) primary antibodies and peroxidase-conjugated secondary antibodies (Amersham Biosciences) before visualization of immunoreactive proteins using ECL kits (Amersham Biosciences). Tubulin levels were used as loading controls.

\section{RNA extraction and Northern blot hybridizations}

Total RNA was isolated from Arabidopsis seedlings using QIAGEN RNeasy Plant Mini Kits (QIAGEN) according to the manu- facturer's instructions. The plasmids, pBA002-3HA and pBA002-6Myc, which carry the $3 \mathrm{HA}$ and $6 \mathrm{Myc}$ DNA fragments, respectively, were digested with AscI/PacI, to generate $0.11-\mathrm{kb}$ $3 \mathrm{HA}$ and $0.22-\mathrm{kb} 6 \mathrm{Myc}$ DNA probes. For Northern blot analysis, $5 \mu \mathrm{g}$ of total RNA was electrophoresed on a $1.2 \%(\mathrm{w} / \mathrm{v})$ agarose gel containing iodoacetamide, and was then blotted onto a nylon membrane (Hybond-N+, Amersham Biosciences). The membrane was hybridized overnight at $65^{\circ} \mathrm{C}$ with either $\alpha^{32}$ P-labeled 3HA DNA probe or $\alpha^{32}$ P-labeled 6Myc DNA probe. The probes were prepared by using a random primer labeling kit (Amersham Biosciences). After overnight hybridization $10.5 \mathrm{M}$ $\mathrm{Na}_{2} \mathrm{PO}_{4}$ at $\mathrm{pH} 7.2 ; 1 \%$ bovine serum albumin; $7 \%$ SDS; 0.5 $\mathrm{mg} / \mathrm{mL}$ denatured salmon sperm DNA), the membrane was washed once ( $1 \times \mathrm{SSC}, 0.1 \%$ SDS) at $65^{\circ} \mathrm{C}$ for $15 \mathrm{~min}$, once $(0.5 \times$ SSC, $0.5 \%$ SDS) at $65^{\circ} \mathrm{C}$ for $15 \mathrm{~min}$, and finally rinsed briefly $(0.1 \times$ SSC, $0.5 \%$ SDS $)$ at $65^{\circ} \mathrm{C}$. The washed membrane was then exposed to X-ray film at $-70^{\circ} \mathrm{C}$.

\section{ß-estradiol, MG132, and cycloheximide treatments}

Ten-day-old double-transgenic Arabidopsis seedlings (35S::HFR1-3HA/XVE::COP1-6Myc) were germinated and grown on selective medium for $10 \mathrm{~d} / 16 \mathrm{~h}$ light $/ 8 \mathrm{~h}$ dark photoperiod) before transfer to liquid MS medium and treatment with MG132 (Calbiochem) plus $\beta$-estradiol (Sigma) or MG132 alone for $16 \mathrm{~h}$ as described previously (Seo et al. 2003). Treated seedlings were harvested for Western and Northern blot analyses. To analyze post-translational events, seedlings treated as above were washed and transferred to fresh MS liquid medium containing $1 \mathrm{mM}$ cycloheximide to block protein synthesis and then exposed to white or FR light. Proteins were extracted at the indicated times and analyzed by Western blotting using anti-HA or anti-Myc (Santa Cruz Biotechnology) antibodies.

\section{Acknowledgments}

We thank Peter Hare for critical reading of the manuscript and Dr Pil-Soon Song for $h$ fr1201. I-C.J. was supported by a postdoctoral fellowship from the Korea Science and Engineering Foundation and, in part, by a Human Frontier Science Program post-doctoral fellowship (LT00309/2004-C). J-Y.Y. was a graduate student on leave from Chung Hsing University, Taiwan. This work was supported by NIH grant GM44640.

\section{References}

Ballesteros, M.L., Bolle, C., Lois, L.M., Moore, J.M., Vielle-Calzada, J.P., Grossniklaus, U., and Chua, N.H. 2001. LAF1, a MYB transcription activator for phytochrome A signaling. Genes \& Dev. 15: 2613-2625.

Bianchi, E., Denti, S., Catena, R., Rossetti, G., Polo, S., Gasparian, S., Putignano, S., Rogge, L., and Pardi, R. 2003. Characterization of human constitutive photomorphogenesis protein 1, a RING finger ubiquitin ligase that interacts with Jun transcription factors and modulates their transcriptional activity. J. Biol. Chem. 278: 19682-19690.

Bolle, C., Koncz, C., and Chua, N.H. 2000. PAT1, a new member of the GRAS family, is involved in phytochrome A signal transduction. Genes \& Dev. 14: 1269-1278.

Clough, S.J. and Bent, A.F. 1998. Floral dip: A simplified method for Agrobacterium-mediated transformation of Arabidopsis thaliana. Plant J. 16: 735-743.

Deng, X.W., Matsui, M., Wei, N., Wagner, D., Chu, A.M., and Feldmann, K.A. 1992. COP1, an Arabidopsis regulatory gene, encodes a protein with both A zinc-binding motif and 
a G- $\beta$ homologous domain. Cell 71: 791-801.

Desnos, T., Puente, P., Whitelam, G.C., and Harberd, N.P. 2001. FHY1: A phytochrome A-specific signal transducer. Genes \& Dev. 15: 2980-2990.

Fairchild, C.D., Schumaker, M.A., and Quail, P.H. 2000. HFR1 encodes an atypical bHLH protein that acts in phytochrome A signal transduction. Genes \& Dev. 14: 2377-2391.

Fankhauser, C. and Chory, J. 2000. RSF1, an Arabidopsis locus implicated in phytochrome A signaling. Plant Physiol. 124: $39-45$

Hardtke, C., Gohda, K., Osterlund, M.T., Oyama, T., Okada, K., and Deng, X.W. 2000. HY5 stability and activity in Arabidopsis is regulated by phosphorylation in its COP1 binding domain. $E M B O$ J. 19: 4997-5006.

Hare, P.D., Møller, S.G., Huang, L.F., and Chua, N.H. 2003 a. LAF3, a novel factor required for normal phytochrome A signaling. Plant Physiol. 133: 1592-1604.

Hare, P.D., Seo, H.S., Yang, J.Y., and Chua, N.H. 2003b. Modulation of sensitivity and selectivity in plant signaling by proteasomal destabilization. Curr. Opin. Plant Biol. 6: 453-462.

Holm, M., Hardtke, C.S., Gaudet, R., and Deng, X.W. 2001a. Identification of a structural motif that confers specific interaction with the WD40 repeat domain of Arabidopsis COP1. EMBO I. 20: 118-127.

Holm, M., Ma, L.G., Qu, L.J., and Deng, X.W. 2001b. Two interacting bZIP proteins are direct targets of COP1-mediated control of light-dependent gene expression in Arabidopsis. Genes \& Dev. 16: 1247-1259.

Hsieh, H.L., Okamoto, H., Wang, M., Ang, L.H., Matsui, M., Goodman,H., and Deng, X.W. 2000. FIN219, an auxin-regulated gene, defines a link between phytochrome A and the downstream regulator COP1 in light control of Arabidopsis development. Genes \& Dev. 14: 1958-1970.

Hudson, M., Ringli, C., Boylan, M.T., and Quail, P.H. 1999. The FAR1 locus encodes a novel nuclear protein specific to phytochrome A signaling. Genes \& Dev. 13: 2017-2027.

Kim, Y.M., Woo, J.C., Song, P.S., and Soh, M.S. 2002. HFR1, a phytochrome A signaling component, acts in a separate pathway from HY5, downstream of COP1 in Arabidopsis thaliana. Plant J. 30: 711-719.

Kost, B., Spielhofer, P., and Chua, N.H. 1998. A GFP-mouse talin fusion protein labels plant actin filaments in vivo and visualizes the actin cytoskeleton in growing pollen tubes. Plant J. 16: 393-401.

Lopez-Molina, L., Mongrand, S., Kinoshita, N., and Chua, N.H. 2003. AFP is a novel negative regulator of ABA signaling that promotes ABI5 protein degradation. Genes \& Dev. 17: 410418.

Møller, S.G., Kunkel, T., and Chua, N.H. 2001. A plastidic ABC protein involved in intercompartmental communication of light signaling. Genes \& Dev. 15: 90-103.

Nagy, F. and Schafer, E. 2002. Phytochromes control photomorphogenesis by differentially regulated, interacting signalling pathways in higher plants. Annu. Rev. Plant Biol. 53: 329355.

Neff, M.M., Frankhauser, C., and Chory, J. 2000. Light: An indicator of time and place. Genes \& Dev. 14: 257-271.

Osterlund, M.T., Hardtke, C.S., Wei, N., and Deng, X.W. 2000. Targeted destabilization of HY5 during light-regulated development of Arabidopsis. Nature 405: 462-467.

Oyama, T., Shimura, Y., and Okada, K. 1997. The Arabidopsis HY5 gene encodes a bZIP protein that regulates stimulusinduced development of root and hypocotyl. Genes \& Dev. 11: 2983-2995.

Quail, P.H. 2002. Phytochrome photosensory signaling networks. Nat. Rev. Mol. Cell Biol. 3: 85-93.
Saijo, Y., Sullivan, J.A., Wang, H., Yang, J., Shen, Y., Rubio, V., Ma, L., Hoecker, U., and Deng, X.W. 2003. The COP1-SPA1 interaction defines a critical step in phytochrome A-mediated regulation of HY5 activity. Genes \& Dev. 17: 2642 2647.

Seo, H.S., Yang, J.Y., Ishikawa, M., Bolle, C., Ballesteros, M.L., and Chua, N.H. 2003. LAF1 ubiquitination by COP1 controls photomorphogenesis and is stimulated by SPA1. $\mathrm{Na}$ ture 26: 995-999.

Seo, H.S., Watanabe, E., Tokutomi, S., Nagatani, A., and Chua, N.H. 2004. Photoreceptor ubiquitination by COP1 E3 ligase desensitizes phytochrome A signaling. Genes \& Dev. 18: 617-622.

Soh, M.S., Kim, Y.M., Han, S.J., and Song, P.S. 2000. REP1, a basic helix-loop-helix protein, is required for a branch pathway of phytochrome A signaling in Arabidopsis. Plant Cell 12: 2061-2074.

von Arnim, A.G. and Deng, X.W. 1994. Light inactivation of Arabidopsis photomorphogenic repressor COP1 involves a cell-specific regulation of its nucleocytoplasmic partitioning. Cell 79: 1035-1045.

Wang, H. and Deng, X.W. 2002. Arabidopsis FHY3 defines a key phytochrome A signaling component directly interacting with its homologous partner FAR1. EMBO J. 21: 1339-1349.

Xie, Q., Frugis, G., Colgan, D., and Chua, N.H. 2000. Arabidopsis NAC1 transduces auxin signal downstream of TIR1 to promote lateral root development. Genes \& Dev. 14: 30243036.

Xie, Q., Guo, H.-S., Dallman, G., Fang, S., Weissman, A. M., and Chua, N.H. 2002. SINAT5 promotes ubiquitin-related degradation of $\mathrm{NACl}$ to attenuate auxin signals. Nature 419: $167-170$.

Yang, K.Y., Kim, Y.M., Lee, S., Song, P.S., and Soh, M.S. 2003. Overexpression of a mutant basic helix-loop-helix protein HFR1, HFR1- $\Delta$ N105, activates a branch pathway of light signaling in Arabidopsis. Plant Physiol. 133: 1630-1642.

Zeidler, M., Bolle, C., and Chua, N.H. 2001. The phytochrome A specific signaling component PAT3 is a positive regulator of Arabidopsis photomorphogenesis. Plant Cell Physiol. 42: 1193-1200.

Zuo, J., Niu, Q.W., and Chua, N.H. 2000. An estrogen receptorbased transactivator XVE mediates highly inducible gene expression in transgenic plants. Plant J. 24: 265-273. 


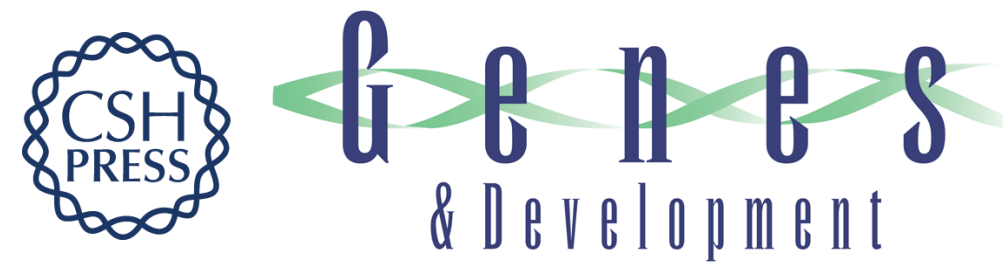

\section{HFR1 is targeted by COP1 E3 ligase for post-translational proteolysis during phytochrome A signaling}

In-Cheol Jang, Jun-Yi Yang, Hak Soo Seo, et al.

Genes Dev. 2005, 19:

Access the most recent version at doi:10.1101/gad.1247205

References This article cites 34 articles, 21 of which can be accessed free at: http://genesdev.cshlp.org/content/19/5/593.full.html\#ref-list-1

License

Email Alerting

Receive free email alerts when new articles cite this article - sign up in the box at the top Service right corner of the article or click here.

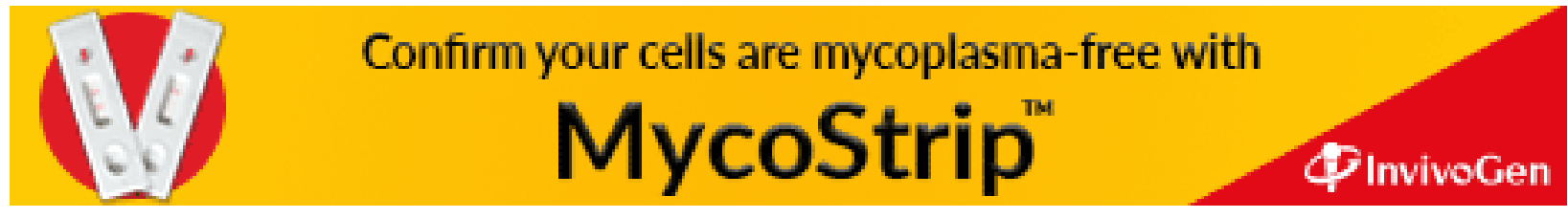

